\title{
Delayed Dysphagia After Removal of Foreign Bodies Near Upper Esophageal Sphincter
}

\author{
Jung Ho Park
}

Department of Internal Medicine, Sungkyunkwan University College of Medicine, Kangbuk Samsung Hospital, Seoul, Korea

A 70-year-old male presented to the emergency department with sudden onset of dysphagia. He had medical history of hypertension for 10 years and cerebral infarction in the territory of the middle cerebral artery 7 year ago. Lateral neck radiography revealed foreign bodies in the upper esophagus (Fig. 1A) and an upper endoscopy was performed. Two seashells with sharp edge were impacted near the upper esophageal sphincter and they were removed using hard straight cap and grasping forceps (Fig. 1B).

Although white blood cell was elevated and small laceration with periesophageal inflammation was suspected at CT scan, his condition improved with non-operative management including broad spectrum antibiotics. However, dysphagia continued for 10 days after removal of foreign bodies. Modified barium swallowing showed large volume of valecular residue with tracheal aspiration despite improvement of inflammation on follow-up CT scan. High-resolution impedance manometry (HRIM) was performed to rule out esophageal motility disorder. HRIM revealed a failure of upper esophageal sphincter relaxation with impaired
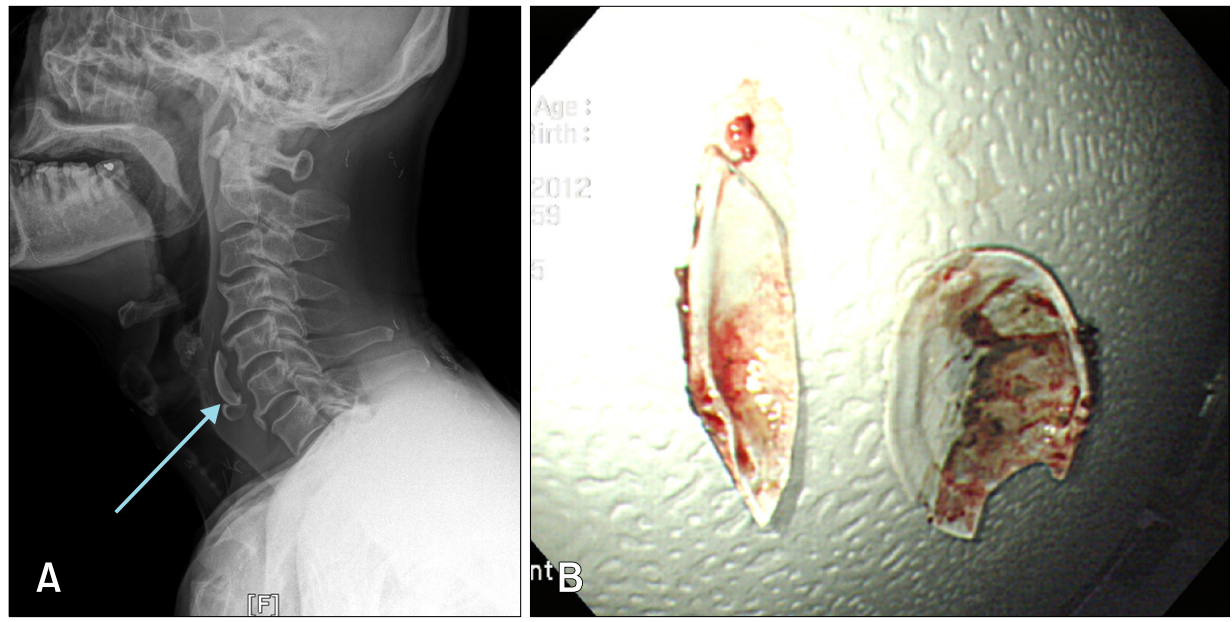

Figure 1. Radiograph of a 70-year-old man who presented with dysphagia. Foreign bodies were lodged in the upper esophagus (blue arrow) (A) and 2 seashells were removed with endoscopic grasping forceps (B).

Received: September 10, 2013 Revised: September 17, 2013 Accepted: September 25, 2013

(c) This is an Open Access article distributed under the terms of the Creative Commons Attribution Non-Commercial License (http://creativecommons. org/licenses/by-nc/3.0) which permits unrestricted non-commercial use, distribution, and reproduction in any medium, provided the original work is properly cited.

*Correspondence: Jung Ho Park, MD

Department of Medicine, Sungkyunkwan University School of Medicine, Kangbuk Samsung Hospital, 29, Saemunan-ro, Jongro-Ku, Seoul 110-746, Korea

Tel: +82-2-2001-2059, Fax: +82-2-2001-2485, E-mail: jungho3.park@samsung.com

Financial support: None.

Conflicts of interest: None. 


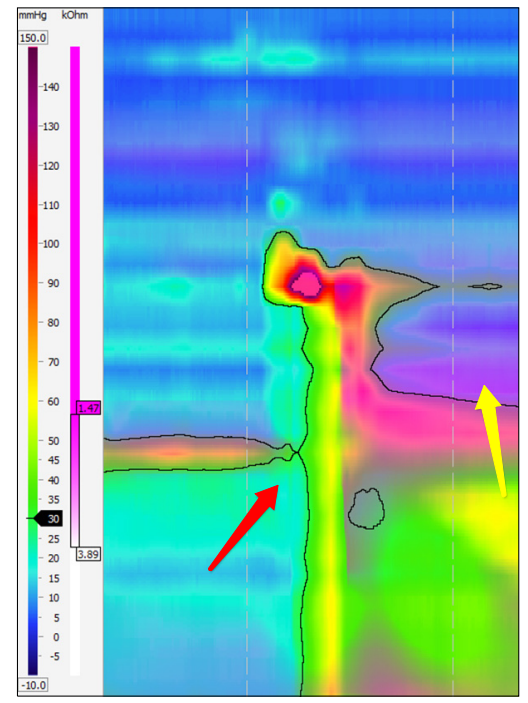

Figure 2. High-resolution impedance manometry (HRIM) findings with $30 \mathrm{mmHg}$ isobaric contour. HRIM presents a failure of upper esophageal sphincter relaxation (red arrow) with impaired bolus transit and intrapharyngeal saline pooling (yellow arrow). bolus transit (Fig. 2). Two days later, he refused to receive further treatment and was discharged.When he visited outpatient clinic 4 days after discharge, dysphagia was improved and he could take soft diet.

Recently, HRIM system was used to develop the clinical parameters of deglutitive upper esophageal sphincter relaxation and normal range values were acquired from healthy volunteers. ${ }^{1}$ In the near future, more accurate diagnosis of dysphagia might be possible based on the objective manometric findings in swallowing difficulty.

\section{Reference}

1. Ghosh S, Pandolfino J, Zhang Q, Jarosz A, Kahrilas P. Deglutitive upper esophageal sphincter relaxation: a study of 75 volunteer subjects using solid-state high-resolution manometry. Am J Physiol Gastrointest Liver Physiol 2006;291:G525-G531. 